\title{
THE UNUSUAL BOX-SHAPED ELLIPTICAL(?) GALAXY IC 3370
}

\author{
Brian Jarvis \\ Geneva Observatory \\ CH-1290 Sauverny \\ Switzerland
}

ABSTRACT. Photometic and kinematic observations are presented for the boxshaped "elliptical" galaxy IC 3370, that show that this galaxy has characteristics more akin to an S0 galaxy than an elliptical and should be classified S0pec.

\section{INTRODUCTION}

The existence of a significant proportion ( $\simeq 1$ pércent) of disk galaxies which possess box- or peanut-shaped bulges is now clear (Jarvis, 1986). However, until recently, similar morphologies in elliptical galaxies have gone unreported. IC 3370 is a galaxy classified as an elliptical in the RC2 and RSA catalogues but shows a strong box shape. Recent attempts to model disk galaxy bulges with box- or peanut-shapes have required large amounts of rotation $\left(V_{m} / \sigma_{0}>1.0\right.$, May, van Albada and Norman 1985; Binney and Petrou 1985). However, most bright elliptical galaxies (say $M_{B}<-21.0$ ) rotate slowly (Davies et al, 1983). Hence, any bright "elliptical" galaxy (i.e. no disk) found to have a box or peanut shape with cylindrical rotation, would be unusual indeed for the reasons mentioned above.For these reasons, the bright box-shaped southern "elliptical" galaxy IC 3370, promised to be an interesting study.

\section{OBSERVATIONS and RESULTS}

Fig. 1 shows the isophotes of IC 3370 in the Johnson B band taken from a CCD image. The $\mathrm{V}$ and $\mathrm{R}$ isophotal maps are similar. The box shape is very noticeable over more than 3.5 magnitudes of surface brightness to the lowest surface brightness measured $\left(\mu_{B}=25.0\right.$ mag.arcsec $\left.{ }^{-2}\right)$ and is strongly suggestive that it extends much further. The minor axis luminosity profile (not shown because of space restrictions) closely follows the de Vaucouleurs $r^{1 / 4}$ law. There is convincing evidence that IC 3370 is an S0 galaxy and not an elliptical since the major axis luminosity profiles, color profiles and fitted ellipses to the isophotes in all colors show a faint stellar disk, visible to a distance of about $15^{\prime \prime}$ on both sides of the nucleus. Fig. 3 shows the isophotes from the central $20^{\prime \prime}$ of IC 3370 in R (least affected by dust) with fitted ellipses. Clear evidence is seen for an excess of light on the major axes of these 
ellipses indicative of a stellar disk embedded within a much more luminous bulge. Further support is provided by the kinematical observations shown in Fig. 2. The maximum rotational velocity on the major axis is $V_{m}=98 \pm 15 \mathrm{~km} . \mathrm{s}^{-1}$, more akin to S0 galaxies than ellipticals. Furthermore, IC 3370 shows strong cylindrical rotation, unlike any elliptical galaxy known at present. The central velocity dispersion was measured to be $\sigma_{0}=220 \pm 20 \mathrm{~km} . \mathrm{s}^{-1}$, giving $V_{m} / \sigma_{0}=0.45$, a value in good agreement with theoretical models of oblate models supported by rotation alone and greater on average than ellipticals of similar luminosity $\left(M_{B}=-22.2\right.$ assuming a distance of $\left.54.3 \mathrm{Mpc}\left(H_{0}=50 \mathrm{~km} \cdot \mathrm{s}^{-1} \cdot \mathrm{Mpc}^{-1}\right)\right)$ and observed flattening.

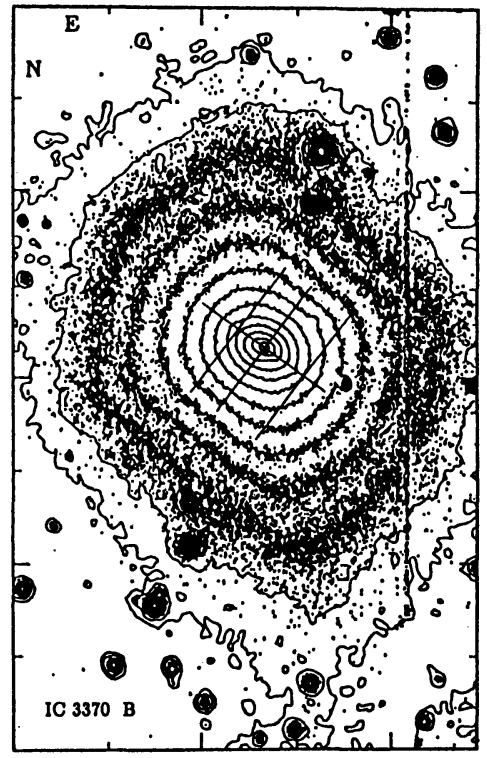

Fig.1 (Top left) Isophotal contour map of IC 3370 in B. The contour interval is 0.5 mag. $\operatorname{arcsec}^{-2}$ with the faintest contour at $\mu_{B}=25.0$ mag. $\operatorname{arcsec}^{-2}$.

Fig.2 (Top right) Rotation curves of IC 3370 along the major axis (top) and perpendicular to the major axis (bottom) but offset $10^{\prime \prime}$ from the minor axis.

Fig.3 (Bottom right) Fitted ellipses to inner 20" of IC 3370 in $R$. Note the disk shown by an excess of light on the major axes of the ellipses.

\section{REFERENCES}

Binney, J., and Petrou, M., 1985, M.N.R.A.S.,214, 449.

Davies, R.L., Efstathiou, G., Fall, S.M., Illingworth, G., and Schechter, P.L., 1983, Ap.J.,266,41.

Jarvis, B.J., 1986, A.J.,91, 65.

May, A., van Albada, T. S., and Norman, C. A., 1985. M.N.R.A.S., $214,131$. 